\title{
Erythropoiétines et dopage : utilisations actuelles, nouvelles perspectives et méthodes de détection
}

\section{Erythropoietins and doping : uses, new perspectives and detection methods. A review}

\begin{abstract}
Aurélie GAUDARD ${ }^{\bullet(2)}$, Emmanuelle VARLET-MARIE $^{\bullet(2)}$, Michel AUDRAN ${ }^{(1)}$,
Françoise BRESSOLLE*(2)
\end{abstract}

(1) Laboratoire de Biophysique, Faculté de Pharmacie, Université Montpellier I - FRANCE

(2) Laboratoire de Pharmacocinétique Clinique, Faculté de Pharmacie, Université Montpellier I - FRANCE

* Auteur à qui adresser la correspondance : Dr Françoise BRESSOLLE, Laboratoire de Pharmacocinétique Clinique, Faculté de Pharmacie - BP 14491 - Université de Montpellier I - 34093 MONTPELLIER Cedex 5 - FRANCE Tél : (33) 467548075 - Fax : (33) 467548075 - E-mail : FBressolle@aol.com A.G. et E.V.M. ont contribué de manière équivalente à ce travail et devraient être considérées comme premier auteur

(Reçu le 20 juillet 2002 ; accepté le 6 septembre 2002)

\section{$R \hat{E} S U M E ́$}

L'érythropoïétine humaine recombinante (alpha et bêta) est utilisée depuis la fin des années 80 par les athlètes dans le but d'augmenter le transport d'oxygène, le pouvoir aérobie, et de ce fait la performance. D'autres molécules apparentées comme la darbépoïétine ont été détournées de leur usage médical à des fins de dopage par certains sportifs. Nous nous proposons, tout d'abord, de présenter les formes d'érythropoiétine existantes ainsi que toutes les molécules et méthodes susceptibles, à plus ou moins long terme, d'augmenter la production d'érythropoiétine ou de mimer son action. Nous exposerons, ensuite, les méthodes directes et indirectes de dépistage d'un dopage à l'érythropoïétine. Enfin, nous discuterons la pertinence de leur utilisation et leur possible application au dépistage d'un dopage par de nouvelles formes d'érythropoiétine ou par des molécules mimant son action.

\section{MOTS-CLÉS}

Dopage, méthodes de détection, érythropoiétines, mimétiques de l'érythropoiétine, génétique.

\section{SUMMARY}

Recombinant human erythropoietin (alfa and beta rHuEpo) is used by some endurance athletes to increase oxygen transport and aerobic power in an attempt to improve endurance capacity and recovery during competition. Other forms of erythropoietin such as darbepoietin are used by athletes in a purpose of doping. First, we review all forms of erythropoietins and methods susceptible of increasing erythropoietin concentration or replacing it. Then, we review all direct and indirect methods to detect erythropoietin abuse. To conclude, we discussed the pertinency of these methods and their potential application to detect new erythropoietins or mimetics.

\section{KEY-WORDS}

Doping, detection methods, erythropoietins, erythropoietin mimetics, genetic. 


\section{Introduction}

Grâce aux progrès du génie génétique, l'érythropoiéti-

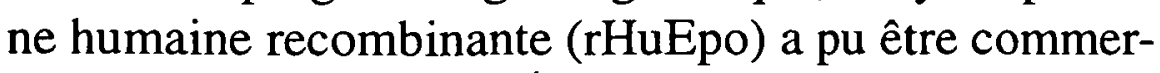
cialisée dès 1986 aux États-Unis. Destinées initialement à traiter le anémies des insuffisants rénaux chroniques, les érythropoiétines alpha et bêta ont progressivement été utilisées pour traiter d'autres anémies : chez le sidéen, le prématuré, le cancéreux, etc... En raison de leur capacité à augmenter l'oxygénation des tissus, ces molécules ont probablement été détournées dès la fin des années 80 par les sportifs d'endurance dans le but d'améliorer leurs performances. Aujourd'hui, de nouvelles érythropoiétines apparaissent -érythropoiétines oméga, delta et darbépoiétine- et de nombreuses recherches sont conduites dans le but de trouver des mimétiques de l'érythropoiétine ou dans le but de modifier la génétique humaine afin d'augmenter la production de l'hormone. Récemment, des implants cellulaires produisant de l'érythropoiétine humaine ont été implantés chez des patients. Les résultats obtenus semblent prometteurs. Il est probable que ces travaux intéressent déjà certains acteurs du monde sportif... Après avoir détaillé le mécanisme d'action de l'érythropoiétine et exposé ses utilisations légales ou illégales, nous présenterons les produits existants sur le marché, les molécules en développement pré-clinique ou clinique ainsi que les voies de recherche prometteuses. Nous répertorierons ensuite les méthodes de détection d'un dopage à l'érythropoïétine. Ces dernières regroupent : 1) les preuves directes d'administration de la rHuEpo et 2) les paramètres indirects de stimulation de l'érythropoièse. En conclusion, nous tenterons de définir les méthodes qui semblent les plus appropriées et les plus prometteuses pour le contrôle anti-dopage. Nous discuterons de la pertinence de ces méthodes pour détecter les nouvelles molécules et techniques susceptibles d'arriver sur le marché dans les mois ou années à venir et d'être détournées pour un usage sportif.

\section{Structure et mécanisme d'action de l'érythropoiétine}

\section{Structure :}

L'érythropoiétine humaine est principalement produite par le rein (1) et faiblement par le foie $(2,3)$. Le gène de l'érythropoiétine code une protéine de 193 acides aminés. Le clivage des 27 acides aminés de la séquence leader conduit à une protéine de 166 acides aminés. La perte de l'arginine en position 166 après la mise en circulation de l'hormone conduit à la forme active de l'érythropoïétine comportant donc 165 acides aminés (4).
L'érythropoiétine circulante est une glycoprotéine de $30,4 \mathrm{kDa}$. Cette forme est glycosylée à $39 \%$. La grande proportion de polysaccharides rend la détermination de structure particulièrement difficile. La partie polysaccharidique de la glycoprotéine a un effet sur sa stabilité et sa solubilité, mais pas sur l'activité in vitro (5). Quatre cystéines permettent la formation de 2 ponts disulfures en positions 29-33 et en 7-161, indispensables à l'activité biologique. Si les structures primaire et secondaire sont connues, la structure tertiaire de l'érythropoiétine reste à déterminer. Un modèle composé de quatre hélices alpha anti-parallèles liées par des anses peptidiques de longueurs variables a été proposé en 1993 par Boissel et al. (6).

\section{Mécanisme d'action :}

L'érythropoiétine se fixe sur un récepteur spécifique induisant le développement des cellules immatures de la lignée érythroide. L'érythropoiétine agit peu sur les BFU-E (Burst Forming Unit Erythroblast). Le nombre de récepteurs de l'érythropoiétine est faible à la surface des BFU-E. Il augmente progressivement avec la maturation des cellules. Le nombre maximum de récepteurs est obtenu au bout de 10 à 21 jours avec la différenciation des BFU-E en CFU-E (Colony Forming Unit Erythroblast). Huit molécules d'érythropoiétine par cellule suffisent à induire la différenciation des CFU-E en érythroblastes en 5 à 8 jours. En l'absence d'érythropoiétine, les CFU-E meurent par apoptose (7). Les érythroblastes se transforment ensuite en érythrocytes qui passent dans la circulation sanguine. Grâce à l'hémoglobine, ils assurent l'oxygénation tissulaire et musculaire. Sous l'action de l'érythropoiétine, on note une augmentation du transport et de la délivrance en oxygène $\left(\mathrm{O}_{2}\right)$ dans l'organisme. La quantité d' $\mathrm{O}_{2}$ disponible exerce alors un rétro-contrôle négatif sur la production d'érythropoiétine. L'action de l'érythropoiétine s'étend aussi à d'autres niveaux de l'érythropoïse : elle augmente la fréquence mitotique des CFUE, accroît le nombre d'ARNm de l'hémoglobine, diminue le temps de transit médullaire, accélérant la sortie des réticulocytes de la moelle (8). Le taux basal d'érythropoiétine dans le sérum chez l'homme sain est de 13 à $28 \mathrm{mUI} / \mathrm{ml}$. Certains auteurs ont observé des variations diurnes des concentrations en érythropoiétine endogène $(9,10,11)$ tandis que d'autres n'ont pas trouvé de variations $(12,13,14)$.

\section{Erythropoïétines et mimé- tiques (Figure 1)}

\section{Erythropoïétines alpha et bêta}

L'érythropoïétine a été le premier facteur de croissance 


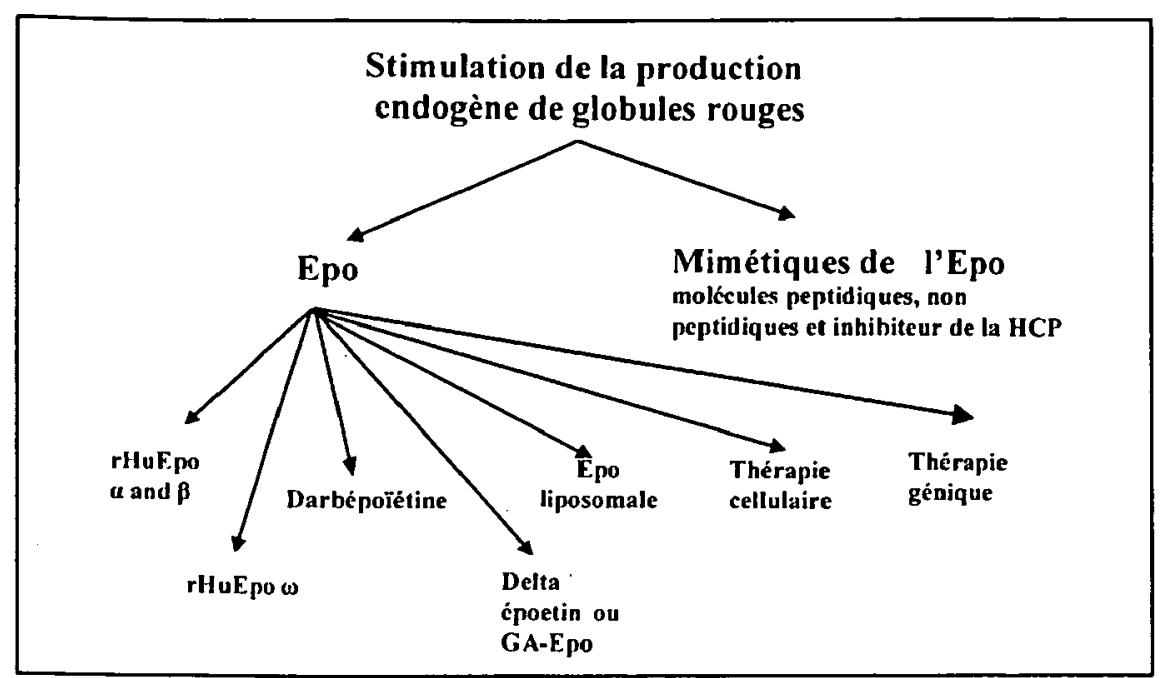

Figure 1 : Molécules et méthodes susceptibles de stimuler la production de globules rouges. (HCP:Phosphatase des cellules hématopoïétiques).

hématopoiétique obtenu par génie génétique. En 1985, deux ans après le clonage du gène, elle a été produite en quantité suffisante pour des essais cliniques. Deux érythropoiétines recombinantes ont été produites sur cellules ovariennes de hamster chinois : la rHuEpo alpha et la rHuEpo bêta. La première société impliquée dans le développement de la rHuEpo a été Amgen qui a mis sur le marché la première érythropoïétine humaine recombinante alpha, l'Eprex ${ }^{\circledR}$, aux États-Unis en 1986. La protéine recombinante a été mise sur le marché en France en 1988. La société Janssen-Cilag commercialise aujourd'hui en France l'érythropoïétine alpha sous le nom d'Eprex ${ }^{\circledR}$ et le laboratoire Roche l'érythropoïetine bêta sous le nom de Neorecormon ${ }^{\circledR}$. La rHuEpo a d'abord été utilisée dans le traitement de l'anémie de l'insuffisance rénale chez le malade adulte hémodialysé et en prédialyse. L'indication a été ensuite étendue au traitement d'autres types d'anémie : anémie de l'enfant insuffisant rénal, du patient adulte cancéreux traité par chimiothérapie à base de platine, des patients participant à un programme de prélèvement autologue différé, des patients adultes traités par chimiothérapie pour des tumeurs solides, des lymphomes malins ou des myélomes multiples. Dans un cadre expérimental, ou dans d'autres pays, l'usage de l'érythropoïetine peut être élargi à d'autres pathologies : cancers divers (15), sida (16)... L'usage de la rHuEpo (alpha et bêta) a été détournée par le milieu sportif. Dans ce cas, l'administration d'érythropoïétine n'est pas utilisée pour pallier une déficience en globules rouges dans le sang. Elle sert à accroître artificiellement la puissance aérobie des sportifs ( $\mathrm{VO}_{2} \max$.), facteur limitant de la performance physique dans les sports d'endurance tels que le cyclisme, mais aussi la course de fonds, le ski de fond, le marathon, le football, la natation, etc. (17). L'abus d'une substance aussi active que l'érythropoïétine s'accompagne d'effets secondaires graves. L'administration répétée de rHuEpo conduit en effet à une hypertension artérielle et à une hyperviscosité sanguine (18) pouvant entraîner des thromboses vasculaires.

\section{Erythropoiétine oméga}

L'érythropoiétine oméga (époétin oméga) est isolée de cellules rénales de bébés hamster. Il s'agit d'une sialoglycoprotéine présentant une plus faible O-glycosylation et une hydrophilie différente des deux précédentes érythropoïétines ; elle est aussi moins acide.

L'érythropoiétine recombinante oméga est commercialisée sous les noms d'Epomax ${ }^{\circledR}$, d'Epomega ${ }^{\circledR}$ ou d'Hemax-Omega ${ }^{\circledR}$ en Europe de l'Est, en Amérique Latine, en Inde et dans certains pays asiatiques. Elle est disponible en flacons de 2000, 4000 et 8000 unités d'époétin omega dans $1 \mathrm{ml}$ de solution stérile. Elle est utilisée dans l'anémie de l'insuffisant rénal chronique $(19,20)$.

\section{La darbépoiétine ("New Erythroid Stimulating Protein")}

La découverte et le développement de la darbépoïétine ou «New Erythroïd Stimulating Protein» sont liés aux progrès de la recherche concernant les caractéristiques structurales de l'érythropoïetine. La darbépoïétine comporte deux chaînes $\mathrm{N}$-glycosylées supplémentaires comparée aux rHuEpo alpha et bêta : elle comporte 5 chaînes N-glycosylées, alors que l'érythropoïétine native et les rHuEpo alpha et bêta n'en ont que 3. Elle a un poids moléculaire plus élevé et une charge négative supérieure en raison d'un nombre plus important d'acides sialiques. Elle se fixe cependant au même récepteur que l'érythropoïétine et agit de la même façon. Actuellement, la darbépoiétine, commercialisée sous le nom de Aranesp ${ }^{\circledR}$, est utilisée dans le traitement de l'anémie de l'insuffisance rénale chronique. Elle recevra vraisemblablement une autorisation officielle d'utilisation dans le cadre de l'anémie du cancer (21). La différence pharmacocinétique majeure de la darbépoïétine par rapport à la rHuEpo alpha ou bêta est sa demi-vie de 25,3 heures après injection intraveineuse (vs 8,4 heures) et de 48,8 heures après injection souscutanée. L'utilisation de la darbépoiétine alpha permet donc des administrations moins fréquentes pour des effets équivalents à la rHuEpo alpha ou bêta (22). La tolérance de la darbépoiétine est similaire à celle de la rHuEpo alpha (23). Les effets secondaires les plus courants sont l'hypertension, l'hypotension secondaire, les myalgies et les maux de tête (24). La séquence aminée de la darbépoïétine diffère de la séquence de l'érythropoiétine en 5 positions. Il est donc possible que la darbépoïétine soit immunogénique. Cette immunogénicité se retrouve avec tous les produits recombinants. La darbépoïétine semble déjà être entrée dans le monde spor- 
tif : trois athlètes ont été exclus de compétitions lors des Jeux de Salt Lake City suite à la découverte de la molécule dans leurs urines : l'Espagnol Muelegg et les Russes Lazutina and Danilova.

\section{Epoetin delta ou "Gene Activated-Epo" (GA-Epo)}

Des chercheurs ont réussi à produire de l'érythropoiétine grâce à un procédé appelé "gene activation", dans lequel la production de protéines résulte de la régulation d'un gène endogène quiescent dans des cellules humaines. Transkaryotic Therapies, Inc. collabore avec Aventis Pharma pour développer et commercialiser la GA-Epo sous le nom de Dynepo ${ }^{\circledR}$ (25). Dynepo ${ }^{\circledR}$ est la seule érythropoïétine produite sur cellule souche humaine. Les autorités européennes et américaines ont émis un avis favorable pour l'autorisation de Dynepo ${ }^{\circledR}$, solution pour injection (flacons de 2000 UI, 3000 UI, 4000 UI, 10000 UI et seringue pré-remplie 1000 UI, 2000 UI, 3000 UI, 4000 UI, 10000 UI) dans le traitement de l'anémie de l'insuffisant rénal chronique.

\section{rHuEpo liposomale}

L'usage de la rHuEpo est limité aux voies intraveineuses et sous-cutanées. Ces injections sont pénibles, et une voie alternative d'administration est souhaitable. Pour éviter la dégradation par le $\mathrm{pH}$ acide de l'estomac et par les enzymes du tractus gastro-intestinal après administration per os, les liposomes ont été étudiés comme transporteur de molécules $(26,27)$. Cependant, "l'érythropoïétine encapsulée" dans des liposomes a seulement été étudiée chez le rat. Les applications humaines sont encore loin. Dans le futur, les athlètes pourront être intéressés par cette forme d'érythropoïetine. La détection dépendra alors de la nature de l'érythropoïétine encapsulée.

\section{Thérapie cellulaire}

Plusieurs équipes de recherche ont proposé la délivrance d'érythropoïétine par l'implantation de cellules protégées dans des membranes polymériques $(28,29,30)$. Récemment, Aebischer et al. (31) ont mis en place sous la peau de quelques patients un implant contenant des cellules sécrétrices d'Epo. Les résultats semblent prometteurs et des essais cliniques vont être mis en place très prochainement sur une vingtaine de sujets.

\section{Thérapie génique}

Depuis environ sept ans, plusieurs auteurs ont démontré la faisabilité du transfert du gène de l'érythropoiétine dans des modèles de rongeurs et de singes $(32,33)$. Les deux principales approches sont le transfert de gène in vivo ou le transfert de gène ex vivo dans descellules isolées qui sont ensuite transplantées dans l'orga- nisme récepteur. Les applications humaines nécessitent un contrôle de la sécrétion d'érythropoïétine pour assurer l'efficacité biologique et éviter les effets toxiques. Rinsch et al. (34) ont proposé une régulation de la délivrance de l'érythropoiétine en fonction de la pression en oxygène. Quatre autres systèmes de régulation pharmacologique sont en développement : ceux régulés par la tétracycline (35), par le RU486 (36), par le stéroïde ecdysone ou ses analogues (36), et par la rapamycine et ses analogues (37). Récemment, Payen et al. (38) ont proposé une régulation à la fois physiologique (par la pression en oxygène) et pharmacologique (par la tétracycline). L'efficacité des systèmes de régulation a été démontrée chez l'animal mais leur application à l'homme semble plus difficile. Reste à voir à quelle vitesse ces développements auront lieu. Cependant, certains scientifiques envisagent déjà la possibilité d'utiliser la thérapie génique dans un but de dopage $(39,40)$ et s'inquiètent des difficultés à détecter un tel dopage.

\section{Mimétiques de l'érythropoïétine}

Le coût et le manque de confort occasionnés par les traitements classiques à la rHuEpo ont conduit à rechercher à délivrer la protéine par voie orale. Aujourd'hui, personne n'a encore réussi à mettre au point un mimétique actif par voie orale. Cependant, un nombre clé d'étapes ont été atteint dans la recherche de petits agonistes de l'érythropoïétine et une bonne connaissance de la structure biologique du récepteur de l'érythropoiétine est maintenant acquise (41).

Le récepteur de l'érythropoïétine (Epo-R) a été cloné indépendamment par plusieurs équipes dès 1990 (42). Le récepteur humain à l'érythropoïétine possède un domaine permettant la fixation de l'hormone (zone $\mathrm{NH}_{2}$ terminale extracellulaire de 250 acides aminés), une région transmembranaire (résidus 251 à 272) et une zone de transduction du signal (zone - $\mathrm{COOH}$ cytoplasmique, résidus $273-508)(43,44)$. Les mécanismes moléculaires induits par la fixation de l'érythropoïétine sur le récepteur ne sont pas totalement élucidés. Bien qu'aucune tyrosine kinase n'ait été identifiée dans le domaine cytoplasmique du récepteur, la fixation d'érythropoiétine provoque la phosphorylation de résidus tyrosine sur le récepteur lui-même et l'activation d'autres protéines, telles que la JAnus Kinase-2 (JAK2) (45). La kinase impliquée en premier lieu est JAK2 (46). La transduction du signal jusqu'au noyau cellulaire peut suivre plusieurs voies (47), en particulier les voies JAK/STAT et ras/MAP (48). Différents mimétiques ont été mis au point pour mimer l'activation de la cascade de réactions qui suivent la fixation de l'érythropoïétine sur son récepteur : 


\section{- Peptides mimétiques de l'érythropoiétine ("Erythropoietin Mimetic Peptides" ou EMPs)}

Wells et al. (49) ont découvert une famille de peptidomimétiques de l'érythropoïétine comprenant 20 acides aminés. L'un d'entre eux, EMP1, a été isolé et la structure du complexe EMP1-EpoR a été déterminée (50, 51). EMP1 est un peptide cyclique de $2 \mathrm{kDa}$. Il ne présente pas d'homologie de séquence avec l'érythropoïétine native et possède une action érythropoiétine mimétique à la fois in vitro et chez l'animal. Wrighton et al. (50) ont évalué la demi-vie de l'EMP1 chez la souris à 8 heures. Ces auteurs ont montré aussi qu'une séquence de 14 acides aminés sur les 20 originaux était nécessaire à l'activité (-YXCXXGPXTWXCXP- où X représente les positions pouvant être occupées par différents acides aminés). Certains auteurs ont montré que la dimérisation de peptides grâce à des liaisons chimiques (52) ou polymériques (53) augmente l'activité. Johnson et al. (53) ont montré qu'il était possible de convertir certains peptides inactifs en agonistes grâce à la dimérisation.

\section{- Ligands au récepteur de l'érythropoüétine ("Erythropoietin Receptor Binders" ou ERBs)}

McConnell et al. (54) ont mis en évidence une famille de peptidomimétiques cycliques composés de 18 acides aminés et dénommés "Erythropoietin Receptor Binders" (ERBs). Ces peptides présentent la séquence minimale active suivante: -CXXGWVGXCXXW- où $\mathrm{X}$ représente les positions susceptibles d'être occupées par différents acides aminés. Bien qu'aucune caractérisation structurale des ERBS liés à leur récepteur n'ait été effectuée, ces molécules semblent être des agonistes complets. Aucune étude, cependant, n'a été réalisée chez l'animal pour tester in vivo l'activité de ces peptides.

\section{- Peptides dérivés du récepteur de l'érythropoïétine ("Erythropoietin Receptor derived Peptides" ou ERPs)}

Un troisième peptido-mimétique, l'EpoR-derived peptide (ERP), a été décrit par Naranda et al. Sa séquence, linéaire, est dérivée d'une méthode par homologie basée sur un travail avec le récepteur de l'insuline (55). L'ERP active la cascade de signalisation activée par l'érythropoḯtine native et est efficace chez l'animal.

\section{- Mimétiques non peptidiques}

D'autres expériences ont montré que l'Epo-R pouvait être activé par des anticorps qui dimérisent le récepteur (56), par mutations qui permettent à deux récepteurs de l'érythropoïétine de se dimériser de manière covalente par la formation de ponts disulfure (57), et par la protéine virale gp55 qui active l'Epo-R indépendamment de l'érythropoïétine (58). Qureshi et al. (59) ont identi- fié un composé appelé "1" (N-3-[2-(4-biphenyl)-6chloro-5-methyl]indolyl-acetyl-L-lysine methyl ester) en testant un ensemble de composés inhibant la fixation de l'érythropoïétine au récepteur. Huit copies de ce composé organisées en oligomère ont donné le composé appelé " 5 " qui est un agoniste de l'érythropoïétine. Ce composé, cependant, ne peut pas être actif par voie orale en raison de son poids moléculaire de 6400 daltons.

\section{Inhibiteurs de la phosphatase des cel- lules hématopoïétiques (HCP)}

HCP se lie au récepteur phosphorylé de l'érythropoïétine et entraîne un changement conformationnel qui permet de déphosphoryler JAK2. HCP agit comme une régulateur négatif de la cascade de signalisation induite par l'érythropoïétine. HCP représente une cible intéressante pour de petites molécules puisqu'un inhibiteur de l'HCP peut augmenter l'action de l'érythropoïétine. Les mimétiques de l'érythropoïétine et l'inhibiteur de l'HCP peuvent intéresser les athlètes pour augmenter leur performance. Cependant, ces produits sont des xénobiotiques et seront probablement facilement détectables.

\section{Méthodes de détection d'un dopage à l'érythro- poïétine}

Plusieurs méthodes ont été proposées pour détecter la prise de rHuEpo : 1) des techniques directes avec identification de l'érythropoḯtine dans le sang ou les urines, 2) des techniques indirectes utilisant des paramètres sanguins, des marqueurs du métabolisme du fer ou encore la mesure des produits de dégradation du fibrinogène dans l'urine et 3 ) des modèles mathématiques multiparamétriques. Toutes ces techniques ont été mises au point et validées en vue de la détection des rHuEpo alfa et bêta. Le dépistage de l'utilisation illicite d'érythropoiétine oméga ou de darbépoiétine devrait pouvoir bénéficier des mêmes méthodes car leur structure moléculaire est suffisamment proche de celle des rHuEpo alfa et bêta. L'utilisation de la méthode directe d'identification mise au point par Lasne et al. (60) pour la rHuEpo alpha et bêta a d'ailleurs permis d'exclure trois athlètes des compétitions lors des jeux de Salt Lake City 2002 après la mise en évidence dans leurs urines de darbépoïétine. Par contre, l'apparition d'autres nouvelles molécules issues de la recherche clinique (époetin delta, mimétiques de nature peptidique et non peptidique, inhibiteurs de la HCP) ou de nouvelles techniques (implants cellulaires) est plus problé- 
matique. La mise au point et la validation de méthodes de détection adaptées à chaque produit sera sans doute rapidement nécessaire pour certains de ces produits.

\section{Méthodes directes}

\section{- Dosages immunologiques}

Il est possible de détecter l'érythropoiétine dans le sérum grâce à des tests radioimmunométriques $(61,62$, $63,64)$ ou de chimiluminescence (62) ou dans les urines grâce à un test radioimmunométrique (65). Ces méthodes, cependant, ne permettent pas de quantifier spécifiquement la rHuEpo et la concentration obtenue doit être corrigée par le niveau basal en érythropoïétine.

\section{- Electrophorèse}

Contrairement aux dosages immunologiques précédents, l'électrophorèse permet de différencier la rHuEpo de l'érythropoiétine endogène car la molécule de rHuEpo est moins «anionique» que l'érythropoiétine endogène. Wide et al. ont été les premiers à suggérer l'utilisation d'une méthode électrophorétique à la fin des années 80 (66). En 1995, une méthode de détection sérique et urinaire a été mise au point par cette équipe (67). Elle consiste à mesurer la charge médiane des isoformes de l'érythropoïétine par électrophorèse en suspension d'agarose $(10 \%)$ suivie d'une élution fractionnée et d'un dosage radioimmunologique. Les isoformes de la rHuEpo se différencient des isoformes de l'érythropoḯtine naturelle par une mobilité électrophorétique médiane plus faible. Grâce à cette méthode, les auteurs ont pu retrouver l'hormone recombinante dans les urines prélevées 24 à 48 heures après une administration de $20 \mathrm{UI} / \mathrm{kg}$ de rHuEpo.

Malheureusement, cette méthode est assez coûteuse, longue à réaliser et la lecture des résultats obtenus est relativement difficile à exploiter.

La variante urinaire de cette méthode a été améliorée récemment par l'équipe du Laboratoire National de Dépistage du Dopage (L.N.D.D.) français (60) et permet d'obtenir une image claire des migrations électrophorétiques. Plusieurs étapes successives sont nécessaires à la mise en évidence de ces profils. Les concentrations d'érythropoiétine dans l'urine étant physiologiquement très faibles (inférieures à $3 \mathrm{UI} / \mathrm{l}$, soit $25 \mathrm{ng} / \mathrm{l}$ ), l'urine est tout d'abord préparée par un procédé d'ultrafiltration permettant d'éliminer dans le filtrat une partie des constituants urinaires de faible poids moléculaire et de concentrer de 500 à 1000 fois les protéines (dont l'érythropoïétine) de plus haut poids moléculaire dans le rétentat. Ce dernier est ensuite soumis à une focalisation isoélectrique (électrophorèse dans un gra- dient de $\mathrm{pH}$ ) qui permet de séparer chacune des protéines présentes en ses isoformes constitutives. L'érythropoiétine ne représente qu'une très faible partie des protéines focalisées (rapport érythropoiétine/protéines totales de l'ordre de 10-7). Aussi la révélation spécifique de l'érythropoiétine nécessite-t-elle la mise en œuvre d'un procédé d'immunoblotting particulier appelé "double-blotting" breveté par les Hospices Civils de Lyon (numéro de brevet 2786 273). Les profils isoélectriques sont enfin visualisés grâce à une réaction de chimiluminescence qui provoque l'émission de lumière au niveau de chacune des isoformes de l'érythropoïétine. Cette émission de lumière est quantifiée au moyen d'une caméra et il est ainsi possible d'évaluer les intensités relatives de chacune des isoformes constitutives d'un profil. L'érythropoïétine endogène est caractérisée par un point isoélectrique (pI) compris entre $\mathrm{pH} 3,92$ et 4,42. Les érythropoiétines recombinantes alpha et bêta ont des $\mathrm{pI}$ compris entre $\mathrm{pH} 4,42$ et 5,11. Il apparaît ainsi que les profils des érythopoiétines recombinantes alpha et bêta sont très proches (avec seulement une isoforme basique supplémentaire dans le cas de la préparation bêta) mais par contre nettement différents de celui de l'érythropoïétine naturelle urinaire constitué d'une majorité d'isoformes plus acides. Cette différence constatée au niveau des profils isoélectriques est le reflet d'une divergence dans certaines modifications post traductionnelles de l'hormone lors de la synthèse en particulier au niveau de sa sialoglycosylation.

\section{- Chromatographie liquide à haute perfor- mance (CLHP)}

Cette méthode, proposée par Rivier et al. en 1999 (68), suppose l'ionisation préalable des chaînes latérales glycosylées de l'érythropoïétine. Elle reste cependant à améliorer. En effet, l'influence du polymorphisme moléculaire n'a pas été bien évaluée et la méthode n'a pas été encore validée.

\section{Méthodes indirectes \\ - Évaluation des paramètres sanguins}

Conconi et al. (69) ont mis en évidence des modifications de la morphologie érythrocytaire consécutive à la prise de rHuEpo : augmentation du volume globulaire moyen (ou des macrocytes) et diminution de la concentration corpusculaire en hémoglobine (ou des cellules hypochromes). Selon cette étude, il serait possible de mettre en évidence une prise exogène de rHuEpo par la mesure du rapport entre le volume globulaire et lá teneur corpusculaire en hémoglobine. Le nombre de macrocytes hypochromes augmente avec la durée de 
conservation des échantillons. Aussi, cette technique présentée lors d'un symposium n'a pas été développée. Selon Ekblom (70), la prise de rHuEpo augmente le nombre de globules rouges, le taux d'hémoglobine, de l'hématocrite et du nombre de réticulocytes. L'auteur a cependant nuancé l'augmentation du taux d'hémoglobine, en précisant que ce paramètre est fonction du sujet (notion de variation interindividuelle). D'ailleurs, des résultats contradictoire avec ceux d'Ekblom ont été mis en évidence par d'autres auteurs. Selon Casoni et al. (71), le nombre de globules rouges et le taux d'hémoglobine ne diffèrent pas significativement chez l'athlète traité par rHuEpo et chez le sujet non traité tandis que les valeurs de l'hématocrite restent plus élevées chez le sujet traité. Pour d'autres auteurs $(61,63,64,66)$, ce sont à la fois le taux d'hémoglobine et l'hématocrite qui augmentent en cas de prise de rHuEpo. Ces différences de résultats peuvent néanmoins s'expliquer par les différences de doses administrées et les rythmes d'injection variables dans chaque étude. Dans toutes les études, le nombre de réticulocytes varie significativement pendant le traitement à la rHuEpo $(54,71,72,73,74,75)$. Souillard et al. (64) relèvent une augmentation de la réticulocytose 2 jours après la première administration. Le nombre de réticulocytes reste élevé les 4 jours suivant la dernière injection. Les auteurs de l'étude observent également que 15 jours après l'arrêt du traitement, le nombre de réticulocytes est plus faible chez les sujets traités que dans le groupe contrôle. Cette valeur plus basse peut s'expliquer par la mise en place d'un rétrocontrôle négatif.

\section{- Évaluation des marqueurs du métabolis- me du fer}

Le transport du fer dans le plasma est assuré par la transferrine qui se fixe sur un récepteur membranaire spécifique dit "TfR" (76) captant préférentiellement les molécules de transferrine diferriques (Figure 2). Après fixation de la transferrine sur son récepteur, le complexe récepteur-transferrine subit une endocytose. Une fois internalisé, il fusionne en une vésicule de $\mathrm{pH}$ inférieur à 5,5 ; cette acidification entraîne le relargage du fer de la transferrine par protonation des groupements impliqués dans la fixation du fer à la protéine. Le fer est alors stocké dans la ferritine, protéine qui peut lier plusieurs milliers d'ions ferriques. Au $\mathrm{pH}$ acide de l'endosome, l'apotransferrine reste liée au récepteur. Le récepteur et l'apotransferrine sont ensuite recyclés via les "CURL" ("Compartment of Uncoupling of Receptor and Ligand") vers la membrane plasmique. $\mathrm{Au} \mathrm{pH}$ neutre externe, l'apotransferrine se détache du récepteur et est relarguée dans le plasma alors que le récepteur est recyclé dans la membrane cellulaire (77).
Pratiquement toutes les cellules de l'organisme ont des TfR à leur surface. Chez l'adulte, ces récepteurs sont localisés à $80 \%$ sur les cellules de la moelle érythropoiétique ; le fer participe alors à la synthèse de l'hémoglobine (76). L'affinité d'un tissu pour le fer est étroitement liée au nombre de récepteurs présents sur la membrane cellulaire (entre 50.000 et 500.000 par cellule) (77). La densité des récepteurs à la surface des cellules dépend notamment de leur état de prolifération - par exemple lors de leur stimulation par l'érythropoḯtine - et de la disponibilité du fer, puisqu'un état ferriprive est un puissant stimulateur de la synthèse du récepteur de la transferrine.

Un dérivé soluble du récepteur de la transferrine (sTfR) a été mis en évidence dans le plasma. Ce récepteur soluble est une forme tronquée du récepteur membranaire, ayant perdu ses 100 premiers acides aminés par cassure protéolytique de la portion extra-cellulaire au niveau des deux ponts disulfure (entre Arg-100 et Leu101). Il est composé de deux monomères de sTfr, de 75.000 daltons chacun, liés de manière non covalente entre eux et complexés à la transferrine $(78,79) .80 \%$ des sTfr proviennent de la moelle osseuse où ils sont relâchés par les précurseurs érythroïdes et par les réticulocytes. Le dosage des sTfr permet l'évaluation quantitative de la réponse érythropoïetique à l'érythropoḯtine, bien avant que des changements du nombre de globules rouges puissent être détectés. La concentration sérique des STfR est donc modifiée dans deux circonstances : elle augmente lors d'un déficit en fer et elle varie en fonction du nombre de précurseurs érythroïdes en prolifération et en différenciation.

L'administration de rHuEpo stimule la production érythroide par la moelle. Du fer est incorporé durant la prolifération des cellules de la lignée rouge. Le métabolisme du fer est donc modifié : la concentration des sTfR augmente tandis que celle de la ferritine (fr) diminue dans le sérum, reflétant la mobilisation des stocks de fer. Par conséquent, le rapport sTfR/fr augmente. De nombreuses études ont retrouvé cette augmentation des sTfR (63) et cette diminution de fr $(62,56)$. Selon Birkeland et al. (62), la concentration en sTfR peut être considérée comme un indicateur d'un dopage à la rHuEpo: les concentrations restent significativement élevés une semaine après administration de l'hormone. Bressolle et al. (63) ont mis au point une méthode statistique basée sur la mesure des sTfr pour détecter une prise de rHuEpo. Les valeurs de ce marqueur supérieures au seuil de $10 \mathrm{mg} / \mathrm{l}$ indiquent vraisemblablement un dopage à l'érythropoïétine.

Gareau et al. (80) ont suggéré en premier l'usage du rapport sTfR/fr qui contrairement à l'hématocrite, ne dépend pas de l'hydratation corporelle. Bressolle et al. 
(63) se sont aussi intéressés à ce rapport et ont déterminé une valeur seuil de 403. Les mesures au delà de ce seuil indiquent la prise vraisemblable de rHuEpo avec une probabilité d'erreur de moins de $1 \%$. De nombreux autres auteurs ont démontré l'intérêt du rapport sTfR/fr $(62,72,74)$. Cependant, comme l'indique une étude de Magnani et al.(81), il n'est utilisable qu'en l'absence de supplémentation en fer et aux fortes doses de rHuEpo. Magnani et al. ont en effet mesuré le rapport sTfR/fr pendant un mois sur trois groupes de sujets. Les deux premiers groupes ont reçu $1000 \mathrm{UI} / \mathrm{kg}$ en 5 doses et le troisième $360 \mathrm{UI} / \mathrm{kg}$ en 12 doses. Du fer a été administré au deuxième groupe qui a reçu $1000 \mathrm{UI} / \mathrm{l}$. L'augmentation du rapport $\mathrm{sTfR} / \mathrm{fr}$ a été significative du deuxième au trente-et-unième jour dans le premier groupe, du quatrième au vingt-quatrième jour dans le deuxième et du dix-huitième au vingt-quatrième dans le troisième. Dans le dernier cas, le rapport n'a jamais excédé 250 .

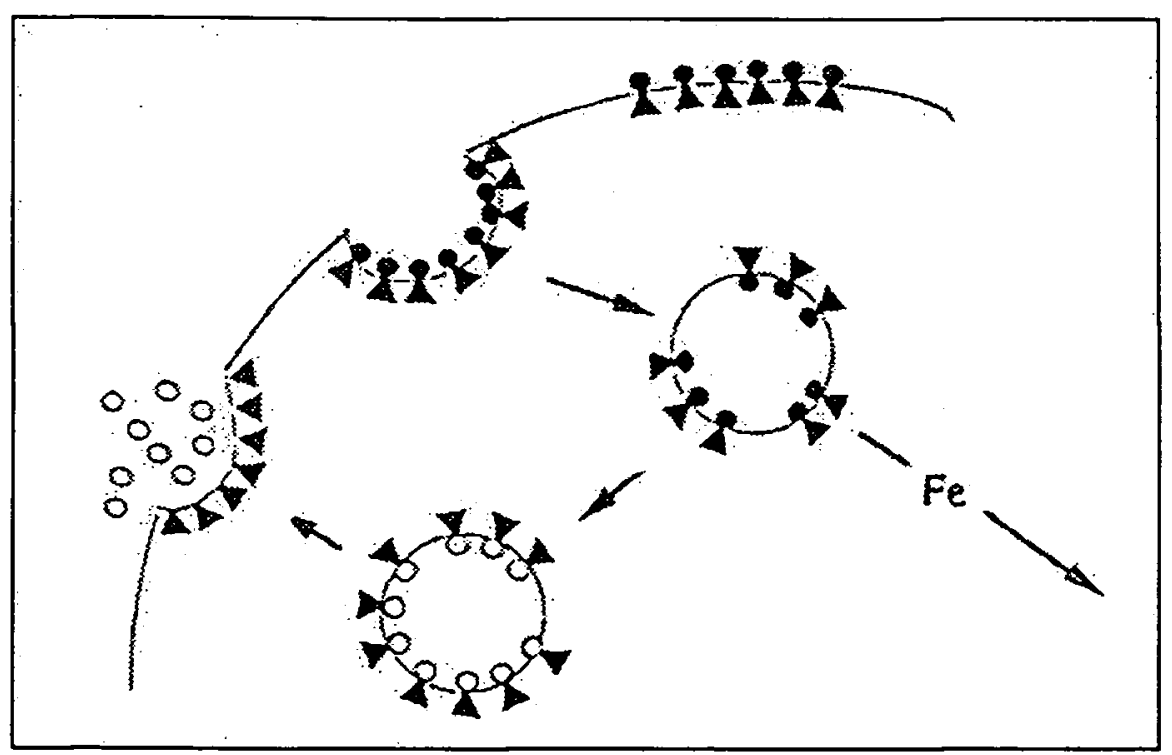

Figure 2 : Cycle intracellulaire de la transferrine et de son récepteur (O) transferrine; ( $\mathbf{\Delta})$ récepteurs à la transferrine; (O) apotransferrine.

\section{- Mesure des produits de dégradation du fibrinogène (PDF)}

L'érythropoïétine, en augmentant le nombre de globules rouges, entraîne une hyperviscosité du sang, responsable de petites réactions locales de coagulation. Ce phénomène correspond à la conversion du fibrinogène en fibrine, catalysée par la thrombine. Pour éviter la thrombose, la plasmine joue un rôle important en permettant la fibrinolyse.

Dans une étude comparative menée chez des patients hémodialysés insuffisants rénaux traités ou non par rHuEpo, il a été montré une augmentation très significative de l'élimination par l'urine des produits de dégradation résultant de la lyse de la fibrine $(82,83)$.
Gareau et al. (75) ont donc pensé que des administrations de rHuEpo pouvaient être décelées dans l'urine en dosant les produits de dégradation de la fibrine. D'autant plus que, même s'il est connu que l'activité fibrinolytique augmente à l'exercice, ce phénomène reste négligeable $(84,85)$. Une étude a donc été menée chez 76 athlètes de haut niveau (86) et a montré une augmentation significative des produits de dégradation de la fibrine chez 13 d'entre eux traduisant pour les auteurs une prise probable de rHuEpo. Cependant, aucune étude ultérieure n'a corroboré les données de ces auteurs. En outre, rien ne permet d'assurer que les $13 \%$ d'athlètes présentant une quantité importante de PDF dans les urines avaient bien pris de la rHuEpo. Enfin, l'analyse reste difficile, chère, nécessite l'utilisation de radio-isotopes et semble donc peu adaptée au contrôle de routine.

\section{Modèles mathématiques multiparamétriques}

Gareau et al. (80) ont été les premiers à suggérer l'utilisation de plusieurs paramètres indirects pour détecter l'usage de rHuEpo. Pour Audran et al. (61), l'augmentation de l'hématocrite associée à un taux de sTfR supérieur à $10 \mathrm{mg} / \mathrm{l}$ et à un rapport sTfr/protéines sériques supérieur à 153 constitue une bonne raison de soupçonner l'usage de rHuEpo. Ces auteurs ont propo-

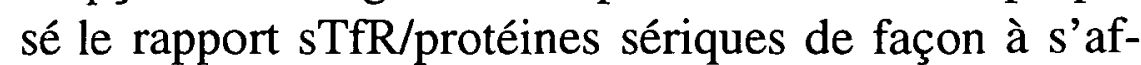
franchir du risque éventuel d'hémoconcentration qui pourrait être à l'origine de faux positifs en élevant artificiellement certains marqueurs comme les sTfR ou l'hématocrite.

Parisotto et al. (72) ont, cependant, montré que le taux de sTfR et le rapport sTfR/protéines sériques sont hautement corrélés $(r=0,96)$ et ne peuvent donc être considérés comme des paramètres indépendants. Les auteurs ont donc décidé d'associer des paramètres directs et indirects. Des athlètes résidant à Sydney et à Pékin ont reçu $50 \mathrm{UI} / \mathrm{kg}$ trois fois par semaine de rHuEpo pendant 25 jours. Les profils sanguins ont été suivis pendant l'administration et jusqu'à 4 semaines après la fin du traitement. L'hématocrite $(\mathrm{Hct})$, l'hématocrite réticulocytaire (ReHct), le pourcentage de macrocytes (\% macro), la concentration sérique en érythropoïetine (Epo) et la concentration en sTfR ont été évalués. Différents modèles incorporant des combinaisons de ces paramètres ont été analysés. Deux modèles ont été proposés : le: «ON-model» qui inclut tous les paramètres et le «OFF-model» qui comprend l'Hct, le ReHct et l'Epo.

Le calcul des scores permettant d'évaluer s'il y a eu 
prise de rHuEpo ou non répond aux formules suivantes :

Score ON-model $=3,721 \mathrm{Hct}+30,45 \mathrm{RetHct}+$ $0,1871 \log _{\mathrm{e}}($ Epo $)+0,1267 \log _{\mathrm{e}}(\mathrm{sTfr})+0,115 \log _{\mathrm{e}}(\%$ macro $+0,1)$

Score OFF-model $=6,149$ Hct $-92,87$ RetHct $0,1463 \log _{\mathrm{e}}$ (Epo)

Le ON-model identifie 94 à $100 \%$ des athlètes ayant reçu de la rHuEpo pendant la phase d'administration et jusqu'à deux semaines après la fin du traitement. Il a été retenu par le Comité International Olympique pour la détection de l'érythropoiétine lors des Jeux de Sydney en 2000. Le OFF-model identifie entre 67 et $72 \%$ des consommateurs de rHuEpo jusqu'à trois semaines après l'arrêt du traitement.

Magnani et al. (81) ont proposé un modèle multiparamétrique prenant en compte l'Hct, le nombre de réticulocytes (Ret), la concentration en sTfR et la quantité d'ARN ${ }_{m}$ de la bêta-globine. La formule adoptée est la suivante :

[Hct (\%) x Ret (\%) x sTfR (nmol/l) x $\mathrm{ARN}_{\mathrm{m}}$ de la bêtaglobine (amol/ $\mu \mathrm{l}$ de sang)] / 100000

La bêta-globine et les sTfR sont des marqueurs sélectifs de l'activité érythroïde. Le dosage de l' $A R N_{m}$ de la bêta-globine est réalisé grâce à la technique de RT-PCR ("Reverse Transcription- Polymerase Chain Reaction") sur le sang total. Cet $\mathrm{ARN}_{\mathrm{m}}$ augmente significativement après un traitement à la rHuEpo. La formule proposée par les auteurs permet de détecter un dopage à l'érythropoḯtine dans $57,5 \%$ des échantillons avec un intervalle de confiance de $99,99 \%$ alors que les méthodes basées sur l'Hct et le rapport sTfR/fr permettent de détecter une prise de rHuEpo dans seulement 7,6\% des échantillons.

Magnani et al. ont également proposé la mesure de l'expression de l' $A R N_{m}$ de la transferrine par RT-PCR sur l'ARN total extrait. Il a été montré que l'augmentation de l'expression de l'ARN $\mathrm{N}_{\mathrm{m}}$ de la transferrine était dépendant du protocole d'administration de la rHuEpo. Aux doses faibles (30 UI/ $\mathrm{kg}$ ), l'augmentation de l'ARN $\mathrm{m}_{\mathrm{m}}$ était plus faible qu'aux fortes doses (200 $\mathrm{UI} / \mathrm{kg}$ ) mais restait toujours détectable pendant la période de traitement. Ces valeurs d' $A R N_{m}$ de la transferrine sont plus faciles et plus rapides à obtenir que celles de l'ARN $\mathrm{m}_{\mathrm{m}}$ de la bêta-globine. Une goutte de sang de $50 \mu \mathrm{L}$ prélevée au bout du doigt suffit à l'analyse. De surcroît, l'inclusion de la quantité d' $A R N_{m}$ de la transferrine dans la formule multiparamétrique permet d'atteindre un pourcentage de détection de 68,1 $\%$. L'étude de Magnani et al. a été menée sur 18 athlètes hors compétition et n'a pas été confirmée par d'autres travaux. Elle nécessite donc d'être étendue à un groupe plus important de sportifs et d'être validée dans des conditions de compétition.

\section{Intérêt et limites des méthodes de dosage}

Les kits commercialisés pour la détection directe de la rHuEpo présentent des sensibilités très variables, ce qui peut représenter un problème pour le contrôle antidopage. Bechensteen et al. (87) ont relevé dès 1993 des valeurs incohérentes de rHuEpo dans différents échantillons testés. En 1999, Marsden et al. (88) ont montré que la précision et la linéarité de différents kits commercialisés étaient encore insatisfaisantes : les mêmes échantillons présentaient des résultats très différents suivant les kits. De plus, ces méthodes ne permettent pas de séparer l'érythropoïétine endogène de l'exogène.

Les méthodes électrophorétiques permettent, en revanche, une telle séparation. De plus, elles présentent l'avantage d'être sûres, de ne pas donner de «faux positifs» et peuvent mettre en évidence un dopage à l'éythropoiétine alfa, bêta, oméga ou à la darbépoiétine dans la mesure où ces molécules ont des différences structurales liées au degré de sialylation des chaînes glucidiques et donc des profils isoélectriques caractéristiques différents. Néanmoins leur usage reste limité à trois jours après la supplémentation en hormone pour les érythropoḯtines alpha et bêta.

Certaines méthodes indirectes ont été employées sur le plasma et l'urine pour détecter le dopage à la rHuEpo. L'Union Cycliste Internationale (UCI) a utilisé l'Hct comme un indicateur d'une prise éventuelle de rHuEpo. L'usage de l'hormone a été suspecté pour des valeurs d'Hct supérieures à $50 \%$ chez l'homme et $47 \%$ chez la femme. La Fédération Internationale de Ski (FIS) a aussi utilisé les valeurs d'hémoglobine. Les seuils tolérés ont été de $175 \mathrm{~g} / 1 \mathrm{chez}$ l'homme et de 155 $\mathrm{g} / \mathrm{l} \mathrm{chez} \mathrm{la} \mathrm{femme.} \mathrm{Cependant,} \mathrm{ces} \mathrm{valeurs} \mathrm{ne} \mathrm{représen-}$ tent pas une preuve de dopage. Des faux positifs ne peuvent pas être exclus. D'une part, certaines maladies (cardiopathies, hémoglobinopathies, polycythémies, hypoxies...) ou les séjours en altitude provoquent une élévation des données hématologiques. D'autre part, des variations individuelles existent et sont connues. L'Hct, par exemple, peut varier physiologiquement entre 42 et 53,6\% $(89,90)$. Enfin, selon Brun et al. (91), l'hématocrite semble influencé par l'entraînement. Un entraînement régulier induit une «autohémodilution» qui diminue la viscosité sanguine. Exclure un athlète d'une compétition sur la base d'une variation d'un paramètre physiologique semble discutable. 
Aussi, aujourd'hui, la seule méthode acceptée par l'UCI n'est plus la valeur d'Hct mais la technique d'électrophorèse. Quand à la FIS, elle prend maintenant en compte plusieurs paramètres : le taux d'hémoglobine, le pourcentage de macrocytes et le nombre de réticulocytes (92).

Les marqueurs du fer doivent être considérés avec précaution. En effet, il n'existe pas vraiment de valeur de référence et les résultats doivent être confirmés par un dosage de l'hormone. Cependant, les sTfr restent les marqueurs indirects les plus efficaces dans le temps, comparativement aux marqueurs directs. Si Parisotto et al. (74) ont démontré que l'éthnicité n'influence pas cinq paramètres différents (Hct, ReHct, \% macro, Epo et sTfR), il ne faut pas oublier qu'une administration de rHuEpo ne peut pas donner une augmentation prédictible de la masse totale d'hémoglobine chez un individu donné. Notamment, le type et la quantité de chaînes de globine synthétisées durant la prolifération érythrocytaire, sont sous contrôle génétique. Certaines populations d'Afrique, d'Asie ou du pourtour méditerranéen sont entre autres bien connues pour développer certaines hémoglobinopathies. L'usage de méthodes multiparamétriques est potentiellement intéressant. Cependant, toutes les méthodes proposées présentent des imperfections. Le ON-modèle proposé par Parisotto et al. conduit à des faux positifs, le OFFmodèle des faux négatifs. La méthode de Magnani et al., quant à elle, reste à valider sur une population d'athlètes plus importante.

\section{Conclusion}

Pratiquement aucune étude n'a été menée à la fois chez l'athlète et chez le sujet sain. Il est donc difficile de savoir : 1) si le sport de haut niveau a vraiment une influence sur les paramètres pharmacocinétiques et pharmacodynamiques ; 2) s'il existe des variations interindividuelles importantes, la notion de détermination de seuils limites autorisés n'aurait plus de sens. Il serait donc intéressant de pouvoir disposer d'un protocole mené dans les mêmes conditions chez l'individu sain et chez le sportif. De surcroît, les doses et les rythmes d'administration utilisés dans les études menées chez le sportif se sont révélés nettement supérieurs à ceux qui se sont avérés être employés sur le terrain, notamment au travers de révélations d'athlètes dopés. Jusqu'à l'affaire Festina, on ignorait en effet complètement les conditions réelles d'utilisation. Ceci restreint encore les données utilisables pour le contrôle anti-dopage. Actuellement, l'utilisation complémentaire de méthodes directes et indirectes chez l'athlète pour détecter un éventuel dopage paraît fondamentale. Si la méthode électrophorétique proposée par Lasne et De Ceaurriz se révèle positive, la prise de produits dopants -tels les érythropoiétines alpha, beta, oméga et la darbépoïétine- est indiscutable. Si cette méthode est «douteuse» un test indirect doit être utilisé pour confirmation du résultat.

La détection de nouvelles molécules comme les peptides mimétiques ou les nouveaux mimétiques non peptidiques capables d'activer le récepteur à l'érythropoiétine, et les inhibiteurs de la HCP ne devrait pas poser de problèmes particuliers dans la mesure où ces produits sont des xénobiotiques. Par contre, le dépistage d'un dopage à l'érythropoḯtine delta semble plus compliqué. Les différences structurales entre l'érythropoiétine endogène et l'érythropoïétine delta seraient minimes voire inexistantes et sa détection de manière directe paraît donc difficile ou même impossible dans l'état actuel des recherches. La détection de l'érythropoïétine produite par les implants cellulaires s'annonce difficile dans la mesure où il s'agit d'une érythropoïétine humaine. Pour finir, certains scientifiques s'inquiètent déjà de l'utilisation à venir de la thérapie génique pour produire de l'érythropoiétine $(93,94)$ : si les chances d'être déclaré positif pour l'athlète sont faibles, celles de nuire à sa santé ne sont pas négligeables, même si le nombre d'accidents suite à un tel dopage reste mal connu. 


\section{Références}

1. Jacobson L.O., Goldwasser E., Fried W., Plzak L. Role of the kidney in erythropoiesis. Nature $1957 ; 179: 633-634$.

2 . Fried $\mathrm{W}$. The liver as a source of extrarenal erythropoietin production. Blood $1972 ; 40: 671-677$.

3. Fisher J.W. Extrarenal erythropoietin production. J. Lab. Clin. Med. 1979 ; 93 : 695-699.

4. Davis J.M., Arakawa T., Strickland T.W., Yphantis D.A. Characterisation of recombinant human erythropoietin produced in Chinese hamster ovary cells. Biochemistry $198 ; 26: 2633-2638$.

5. Narhi L.O., Arakawa T., Aoki K.H., Elmore R., Boone T., Stricland T.W. The effect of carbohydrate on the structure and stability of erythropoietin. J. Biol. Chem. 1991 ; $266: 23022$

6. Boissel J.P., Lee W.R., Presnell S.R., Cohen F.E., Bunn H.F. Erythropoietin structure-function relationships, J. Biol. Chem. 1993 ; 268 : 15983-15993.

7. Koury M.J., Bondurant M.C. The molecular mechanism of erythropoietin action. Eur. J. Biochem. $1992 ; 210$ : 649-663.

8. Pheulpin P. Etude comparée des erythropoiétines humaines natives et recombinantes. Thèse de Pharmacie, Montpellier 1992.

9. Cotes P.M. Immunoreactive erythropoietin in serum. I. Evidence of the validity of the assay method and the physiological relevance of estimates. Br. J. Haematol. 1982 ; $50: 427-438$.

10.Buemi M., Allegra A., Alloisi C. et al. The circadian rhythm of erythropoietin in subjects with pre-terminal uraemia. Clin. Nephrol. $1992 ; 37: 159-160$.

11. Cahan C., Decker M.J., Arnold J.L. et al. Diurnal variations in serum erythropoietin levels in healthy subjects and sleep apnoea patients. J. Appl. Physiol. $1992 ; 72$ : 2112-2117.

12. Adamson J.W., Alexanian R., Martinez C. Erythropoietin excretion in normal man. Blood $1966 ; 28: 354-364$.

13. McKee L.C., Johnson L.E., Lange R.D. Circadian variation in reticulocyte counts and immuno-detectable erythropoietin titters. Proc. Biol. Med. 1974 ; 145 : 12841287.

14. Miller M.E, Garcia J.F., Cohen R.A. et al. Diurnal levels of immunoreactive erythropoietin in normal subjects and subjects with chronic lung disease. Br. J. Haematol. $1981 ; 49: 189-200$.

15. Spivak J.L. Cancer-related anaemia : its causes and characteristics, Semin. Oncol. $1994 ; 21$ : 3-8.

16. Jacobsen M.A., Peiperi L., Volberding P.A. et al. Red cell transfusion therapy for anemic patients with AIDS and ARC : incidence, associated factors and outcome, Transfusion $1990 ; 30: 133-137$.

17. Kickman A.T., Cowan D.A. Peptide hormones and sport : misuse and detection, Br. Med. Bull. 1992 ; 48 : 496-517.

18. Singbart G. Adverse events of erythropoietin in longterm and in acute/short-term treatment, Clin. Invest. $1994 ; 72: 36-43$.
19. Bren A., Kandus A., Varl J. et al. A comparison between epoetin omega and epoetin alfa in the correction of anemia in hemodialysis patients : a prospective, controlled crossover study. Artif. Organs 2002 ; 26(2) : 91-7.

20. Sikole A., Spasovski G., Zafirov D. et al. Epoetin omega for treatment of anaemia in maintenance hemodialysis patients. Clin. Nephrol. 2002 ; 57(3) : 237-245.

21. Ibbotson T., Goa KL. Darbepoietin alfa. Drugs 2001 ; 61(14) : 2097-2104.

22. Macdougall I.C., Gray S.J., Elston O. et al. Pharmacokinetics of Novel Erythropoiesis stimulating protein compared with epoetin alfa in dialysis patients. $\mathbf{J}$. Am. Soc. Nephrol. 1999 ; 10 : 2392-2395.

23. Vanrenterghem Y., Barany P., Mann J. et al. Novel erythropoieis stimulating protein (NESP) maintains haemoglobin $(\mathrm{Hgb})$ in ESRD patients with administered once weekly or once every other week. J. Am. Soc. Nephrol. $1999 ; 10$ : A1365.

24. Nissenson A.R. Novel erythropoiesis stimulating protein for managing the anemia of chronic kidney disease. Am. J. Kidney Dis. 2001 ; 38(6) : 1390-1397.

25. De Bree F. Genomics-based drugs in R\&D the promise of a new era. Genomics-based drugs data report and regenerative. Therapy $2001 ; 1(1): 5-19$.

26. Maitani Y., Hazama M., Tojo Y. et al. Oral admininstration of recombinant human erythropoietin in liposomes in rats: influence of lipid composition and size of liposomes on bioavailability. J. Pharm. Sci. 1996 ; 85 : 440-445.

27. Maitani Y., Moriya H., Shimoda N. et al. Distribution characteristics of entrapped recombinant human erythropoietin in liposomes a,d its intestinal absorption in rats. Int. J. Pharm. $1999 ; 185: 13-22$.

28. Regulier E., Schneider B.L., Deglon N., et al. Continous delivery of human and mouse erythropoietin in mice by genetically engineered polymer encapsulated myoblasts. Gene Ther. 1998 ; 5(8) : 1014-1022.

29. Dalle B., Payen E., Regulier R., et al. Improvement of mouse-thalassemia upon erythropoietin delivery by encapsulated myoblasts. Gene Ther. $1999 ; 6(2): 157-161$.

30. Serguera C., Bohl D., Rolland E., et al. Control of erythropoietin secretion by doxycycline or mifeprostone in mice bearing polymer-encapsulated engineered cell. Hum. Gene Ther. 1999 ; 10(3) : 375-383.

31.www.snf.ch/fr/com/prr/prr_cur_mar19.asp.

32. Kessler P., Podsakoff G., Chen X. et al. Gene delivery to skeletal muscle results in sustained expression and systemic delivery of a therapeutic protein. Proc. Natl. Acad. Sci. USA. 1996 ; 93 : 14082-14087.

33. Zhou S., Murphy J.E., Escobedo J.A. et al. Adeno-associated virus-mediated delivery of erythropoietin leads to sustained elevation of hematocrit in nonhuman primates. Gene Ther. 1998 ; 5 : 665-670.

34. Rinsch C., Régulier E., Déglon N., et al. A gene therapy approach to regulated delivery of erythropoietin as a function of oxygen tension. Hum. Gene Ther. $1997 ; 8$ : 1881-1889. 
35. Seguera C., Bohl D., Rolland E. et al. Control of erythropoietin secretion by doxycycline or mifeprostone in mice bearing polymer-encapsulated engineered cell. Hum. Gene Ther. 1999 ; 10(3) : 375-383.

36. Clakson T. Regulated gene expression systems. Gene Ther $2000 ; 7: 120-125$.

37. Ye X., Rivera V.M., Zoltick P. et al. Regulated delivery of therapeutic proteins after in vivo somatic cell gene transfer. Science $1999 ; 283-292$.

38. Payen E., Bettan M., Henri A., et al. Oxygen tension and a pharmacological switch in the regulation of transgene expression for gene therapy. J Gene Med 2001 ; 3 : 498-504.

39. Adam D. Gene therapy may be up to speed for cheats at 2008 Olympics. Nature $2001 ; 414$ : 569-570.

40. Friedmann T., Koss J.O. Gene transfer and athletics- An impending problem. Mol. Ther. 2001 ; 3(6) : 819-820.

41. Boulay J.L., Paul W.E. Haematopoietin sub-family classification based on size, gene organization and sequence homology. Curr. Biol. 1993 ; 3 : 573-581.

42. Jones S.S., D’Andrea A.D., Haines L.L., Wong G.G. Human erythropietin receptor: cloning, expression, and biologic characterization. Blood $1990 ; 76: 31-35$.

43. Barber D.L., D'Andrea A.D. The erythropoietin receptor and the molecular basis of sigma 1 transduction. Semin. Hematol. 1992 ; 29(4) : 293-304.

44. Winkelmann J.C., Penny L..A., Deaven L.L., Forget B.G., Jenkins R.B. The gene for the human erythropoietin receptor: analysis of the coding sequance and assignment to chromosome 19p. Blood 1990 ; 76 : 24-30.

45. Tanner J.W., Chen W., Young R.L., Longmore G.D., Shaw A.S. The conserved box 1 motif of cytokine receptors is required for association with JAK kinases. J. Biol. Chem. $1995 ; 270: 6523-6530$.

46. Witthuhn B.A., Quelle F.W., Silvennoinen O., Yi T., Tang B., Miura O., Ihle J.N. JAK2 associates with the erythropoietin receptor and is tyrosine phosphorylated and activated following stimulation with erythropoietine. Cell 1993 ; $74: 227-236$.

47. Constantinescu S.N., Ghaffari S., Lodish H.F. The erythopoietin receptor : structure, activation and intracellular signal transduction, TEM $1999 ; 10: 18-23$.

48. Ihle J.N. Cytokine receptor signalling, Nature 1995 ; 377 : 591-594.

49. Wells J.A. Hormone mimicry. Science $1996 ; 273$ : 449-450.

50. Wrighton N.C., Farrell F.X., Chang R. et al. Small peptides as potent mimetics of the protein hormone erythropoietin. Science $1996 ; 273: 458-463$.

51. Livnah O., Stura E.A., Johnson D.L. et al. Functional mimicry of a protein hormone by a peptide agonist: the Epo receptor complex at $2.8 \AA$. Science $1996 ; 273: 464-471$.

52. Wrighton N.C., Balasubramanian P., Barbone F.P. et al. Increased potency of an erythropoietin peptide mimetic through covalent dimerization. Nature Biotechnology $1997 ; 15: 1261-1265$.

53. Johnson D.L., Farrell F.X., Barbone F.P. et al. Identification of a 13 amino acid peptide mimetic of erythropietin and description of amino acids critical for the mimetic activity of EMP1. Biochemistry 1998 ; 37(11) : 3699-3710.
54. McConnell S.J., Dinh T., Le M.H. et al. Isolation of erythropoietin receptor agonist peptides using evolved phage libraries. Biol. Chem. 1998 ; 379 : 1279-1286.

55. Naranda T., Wong K., Kaufman R.I. et al. Activation of erythropoietin receptor in the absence of hormone by a peptide that binds to a domain different from the hormone binding site. Proc. Natl. Acad. Sci. USA 1999 ; 96 : 7569-7574.

56. Elliot S., Lorenzini T., Yanaghira D. et al. Activation of the erythropoietin (EPO) receptor by bivalen $t$ anti-Epo receptor antibodies. J. Biol. Chem. 1996 ; 271 : 2469124697.

57. Yoshimura A., Longmore G., and Lodish H.F. Point mutation in the exoplasmic domain of the erythropoietin receptor resulting in hormone-independent activation and tumorigenicity. Nature $1994 ; 348$ : 647-649.

58.Li J.P., D'Andrea A.D., Lodish H.F. et al. Activation of cell growth by binding of Friend Spleen focus forming gp55 glycoprotein to the erythropoietin receptor. Nature $1990 ; 343: 762-764$.

59.Qureshi S.A., Kim R.M., Konteatis Z. et al. Mimicry of erythropoietin by a nonpeptide molecule. Proc. Natl. Acad. Sci. USA 1999 ; 96(21) : 12156-12161.

60.Lasne F., De Ceaurriz J. Recombinant erythropoietin in urine. Nature $2000 ; 405: 635$.

61.Audran M., Gareau R., Matecki S., Durand F., Chenard C., Sicart M., Marion B., Bressolle F. Effects of erythropoietin administration in training athletes and possible indirect detection $\mathrm{n}$ doping control. Med. Sci. Sports Exerc. $1999 ; 31: 639-645$.

62. Birkeland K., Hemmersbach P. Issues related to blood sampling in athletes for doping control. Sports Med. $1999 ; 28: 25-30$.

63.Bressolle F., Audran M., Gareau R., Baynes R.D., Giudicelli C., Gomeni R. Population pharmacodynamics for monitoring epoetin in athletes. Clin. Drug. Invest. $1997 ; 14: 233-242$.

64. Souillard A., Audran M., Bressolle F., Gareau R., Duvallet A., Chanal L. Recombinant human erythropoietin and pharmacodynamic parameters in athletes. Interest of blood sampling for doping control. Br. J. Clin. Pharmacol. 1996 ; 42 : 355-360.

65. Kendall R.G., Chapman C., Hartley A.E., Norfolk D.R. Storage and preparation of samples for erythropoietin radioimmunoassay. Clin. Lab. Haematol. 1991 ; 13 : 189 . 196.

66. Wide L., Bengtsson C., Birgegard G. Circadian Rhythm of erythropoietin in human serum. Brit. J. Haematol. $1989 ; 72: 85-90$.

67. Wide L., Bengtsson C., Berglund B., Ekblom B. Detection in blood and urine of recombinant erythropoietin administered to healthy men. Med. Sci. Sports Exerc. $1995 ; 27: 1569-1576$.

68. Rivier R., Saugy M. Peptide hormones abuse in sport: state of the art in the detection of growth hormone and erythropoietin. Journal of Toxicology. Toxin. Reviews $1999 ; 18: 145-176$.

69. Conconi F., Casoni I., Manfredini F. et al. Detection of erythropoietin administration in sports. Blood Samples in Doping Control Second international symposium on drugs in sports, Lillehamer, 1993 ; 133-140. 
70. Ekblom B. Blood doping and erythropoietin: the effects of variation in haemoglobin concentration and other related factors on physical performance. Am. J. Sports. Med. $1996 ; 24$ : S40-S42.

71. Casoni I., Ricci G., Ballarin E., Borseto C., Grazzi G., Guglielmini C., Manfredi F., Mazzoni G., Patracchini M., De Paoli Vitali E., Rigolin F., Bartalotta S., Franzè G.P., Masotti M., Conconi F. Haematological indices of erythropoietin administration in athletes. Int. J. Sports Med. $1993 ; 14: 307-311$.

72. Parisotto R., Gore C.J., Emslie K.R., Asheden M.J., Brugnara C., Howe C., Martin T., Trout G.T., Hahn A.G. A novel method utilizing markers of altered erythropoiesis for the detection of recombinant human erythropoietin abuse in athletes. Haematologica $2000 ; 85: 564-572$.

73. Parisotto R., Gore C.J., Hahn A.G., Ashenden M.J., Olds T.S., Martin D.T., Pyne D.B., Gawthorn K., Brugnara C. Reticulocyte parameters as potential discriminators of recombinant human erythropoietin abuse in elite athletes. Int. J. Sports Med. $2000 ; 21: 471-479$.

74. Parisotto R., Moutian W., Ashenden M.J., Emslie K.R., Gore C.J., Howe C., Kazlauskas R., Sharpe K., Trout G.J., Xie M., Hahn A.G. Detection of recombinant human erythropoietin abuse in athletes utilizing markers of altered erythropoiesis. Haematologica $2001 ; 86$ : 128137.

75.Russell G., Gore C.J., Ashenden M.J., et al. Effects of prolonged low doses of recombinant human erythropoietin during submaximal and maximal exercise. Eur. J. Appl. Physiol. 2002 ; 86 : 442-446.

76. Beguin Y. The soluble transferring receptor: biological aspects and clinical usefulness as quantitative measure of erythropoiesis. Haematologica $1992 ; 77: 1-10$.

77. Shechter E. Biochimie et biophysique des membranes. Masson éditeur. $1990 ; 331-332$.

78. Shih Y.J., Baynes R.D., Hudson B.G., Flowers C.H., Skikne B.S., Cook J.D. Serum transferrin receptor is a truncated form of tissue receptor. J. Biol. Chem. 1990 ; $265: 19077-19081$.

79. Baynes R.D., Shih Y.J., Hudson B.G., Cook J.D. Production of the serum form of the transferrin receptor by a cell membrane-associated serine protease. Proc. Soc. Exp. Biol. Mol. 1993 ; 204 : 65-69.

80. Gareau R., Audran M., Baynes R., Flowers C., Duvallet A., Senecal L., Brisson G. Erythropoietin abuse in athletes. Nature $1996 ; 380: 113$.

81. Magnani M., Corsi D., Bianchi M., Paiardini M., Galluzzi L., Gargiullo E., Parisi A., Pigozzi F. Identification of blood erythroid markers useful in revealing erythropoietin abuse in athletes. Blood Cells Mol. Dis. $2001 ; 27(3)$ : 559-571.
82. Gareau R., Gagnon M.G., Ayotte C., Chenard C., Brisson G.R. RHuEpo increases urinary excretion of fibrin degradation products in haemodialysed patients. Thromb. Haemost. 1993 ; 70 : 373-374.

83. Szewczuk J., Mazerska M., Malysko J. et al. Increase in fibrinolytic activity after erythropoietin therapy. Thromb. Haemost. $1992 ; 67: 284$.

84. Gareau R., Brisson G.R, Ayotte C., Dube J., Caron C. Erythropoietin doping in athletes : possible detection through measurement of fibrinolytic products. Thromb.Haemost. $1992 ; 68: 481-482$.

85. Davis R.B., Boyd D.G., Kinney M.C., Jones C.C. Effects of exercise and exercise conditioning on blood platelet function. Med. Sci. Sport. Exerc. $1990 ; 22: 49-53$.

86. Gareau R, Brisson G.R., Chenard C., Gagnon M.G., Audran M. Total fibrin and fibrinogen degradation products in urine : a possible probe to detect illicit users of the physical-performance enhancer erythropoietin? Horm. Res. 1995 ; 44 : 189-192.

87. Bechensteen A.G., Lappin T.R., Marsden J., Muggelston D., Cotes P.M. Unreliability in immunoassays of erythropoietin: anomalous estimates with an assay kits. $\mathrm{Br}$. J. Haematol. $1993 ; 83$ : 663-664.

88. Marsden J.T., Sherwood R.A., Peters T.J. Evaluation of six erythropoietin kits. Ann. Clin. Biochem. $1999 ; 36$ : 380-387.

89. Cazzola M. A global strategy for prevention and detection of blood doping with erythropoietin and related drugs. Haematologica $2000 ; 85: 561-563$.

90.Lee G.R., Bithell T.C., Foerster J., Athens W., Lukens J.N. Winthrobe's clinical haematology, 9th edition. Philadelphia, PA: Lea and Febiger, 1993.

91. Brun J.F., Bouchadha C., Chaze D., Aissa Benhaddad A., Micallef J.P., Mercier J. The paradox of hematocrit in exercise physiology: which is the "normal" range from an hemorheologist's viewpoint? Clin. Hemorheology Microcirculation $2000 ; 22: 287-303$.

92. Stray-Gundersen J. Red cell parameters in winter endurance sports. XIVth international on technological innovations in laboratory haematology. Le Corum, Montpellier (France) 9 sept. 2001.

93. Adam D. Gene therapy may be up to speed for cheats at 2008 Olympics. Nature $2001 ; 414$ : 569-570.

94. Friedmann T., Koss J.O. Gene transfer and athletics- An impending problem. Mol. Ther.2001;3(6) : 819-820. 